\title{
An Idiotypic Marker Associated with a Germ-line Encoded $\kappa$ Light Chain Variable Region That Predominates the Vaccine-induced Human Antibody Response to the Haemophilus influenzae b Polysaccharide
}

\author{
Alexander H. Lucas, * Raymond J. Langley," Dan M. Granoff," Moon H. Nahm," Marina Y. Kitamura," and Mitchell G. Scott' \\ ${ }^{*}$ Children's Hospital Oakland Research Institute, Oakland, California 94609; and ${ }^{\ddagger}$ Division of Infectious Diseases, The Edward \\ Mallinckrodt Department of Pediatrics, and ${ }^{\S}$ Division of Laboratory Medicine, Department of Pathology, \\ Washington University School of Medicine, St. Louis, Missouri 63110
}

\begin{abstract}
Human antibodies specific for the Haemophilus influenzae b polysaccharide (Hib PS) frequently express a cross-reactive idiotype (CRI), and commonly utilize a $V_{L}$ region that is the product of the $V_{k}$ II gene A2. To examine further anti-Hib PS $V$ region expression and to determine whether $C R I$ expression is correlated with the VKIIA2 chain, we isolated a monoclonal antibody (MAb) reactive with an idiotypic determinant of antiHib PS antibodies. This MAb inhibited Hib PS binding but did not react with Ig isotypic determinants. The CRI recognized by this MAb, designated HibId-1, was associated with the Hib PS-combining site since the reactivity of the MAb with antiHib PS antibodies could be inhibited by Hib PS. HibId-1 was expressed by 17 of 17 clonally purified and sequence-defined anti-Hib PS antibodies having VאIIA2 $L$ chains. In contrast, 0 of 10 anti-Hib PS antibodies having either V $\lambda$, VkI, or VkIII chains expressed HibId-1. Western blot analysis showed that the MAb anti-CRI reacted with isolated anti-Hib PS VkIIA2 $L$ chains but not with $H$ chains or other $L$ chains, indicating that the HibId-1 determinant is localized to the VkIIA2 chain, and does not require pairing with $\mathrm{H}$ chain for expression.

Anti-Hib PS antibodies bearing HibId-1 were present in at least $85 \%$ of subjects immunized with either free Hib PS or Hib PS coupled to diphtheria toxoid (Hib PS-DT), and comprised on the average $60 \%$ of the total vaccine-induced serum anti-Hib PS. HibId-1 expression was not related to age at vaccination inasmuch as infants, children, and adults had similar distributions of HibId-1-positive anti-Hib PS after vaccination with Hib PS-DT. HibId-1 was expressed at a lower frequency and comprised a smaller fraction of the total anti-Hib PS antibody in adult preimmunization sera as compared to post-Hib PS immunization sera, suggesting that immunization preferentially
\end{abstract}

Some of the results reported here were presented at the joint meeting of the American Society for Biochemistry and Molecular Biology and the American Association of Immunologists, June 1990, New Orleans, LA, and at the Third International Symposium on Epidemiology, Pathogenesis and Prevention of Haemophilus influenzae Disease, September 1990, Veldhoven, The Netherlands.

Dr. Langley's current address is NIH/NIAID, Twinbrook II, Rockville, MD 20852.

Address reprint requests to Dr. Lucas, Children's Hospital Oakland Research Institute, 747 52nd Street, Oakland, CA 94609.

Received for publication 24 May 1991 and in revised form $15 \mathrm{Au}$ gust 1991

\section{J. Clin. Invest.}

(C) The American Society for Clinical Investigation, Inc.

$0021-9738 / 91 / 12 / 1811 / 08 \quad \$ 2.00$

Volume 88, December 1991, 1811-1818 stimulates HibId-1-positive B cells. These data demonstrate that antibodies bearing HibId-1/VkIIA2 comprise a predominant component of the anti-Hib PS response induced by immunization, and that this pattern of $V_{L}$ expression is established early in ontogeny. (J. Clin. Invest. 1991. 88:1811-1818.) Key words: antibody repertoire $\bullet$ carbohydrate $\bullet$ cross-reactive idiotype $\bullet$ vaccine $\bullet$ variable region

\section{Introduction}

Haemophilus influenzae type $\mathrm{b}(\mathrm{Hib})^{1}$ is an encapsulated bacterium that causes serious invasive disease, particularly among young children $(1,2)$. The capsular polysaccharide of $\mathrm{Hib}$ (Hib PS), which consists of repeating units of 3- $\beta$-D-ribose-(1-1)-ribitol-5-phosphate, is a major virulence factor, and antibodies specific for the Hib PS confer protection against Hib disease. Like many bacterial polysaccharides, Hib PS is poorly immunogenic in children less than $2 \mathrm{y}$ of age (3), but conjugation of protein to Hib PS markedly increases its immunogenicity (4, 5). Immunization of infants as young as $2-4$ mo of age with Hib PS conjugate vaccines induces significant levels of serum anti-Hib PS antibody $(6,7)$, and probably results in protective immunity.

While the use of conjugate vaccines offers great promise for the prevention of invasive Hib disease, much still remains to be learned about immunity to Hib PS. For example, there is considerable variability in the antibody responses induced by different Hib PS vaccines. Some individuals develop invasive Hib disease despite vaccination (8), and some conjugate vaccines may be poorly immunogenic or of low efficacy in particular ethnic groups $(9,10)$. Furthermore, the protective ability of human anti-Hib PS antibodies may be variable since differences in antibody functional activity and avidity have been described $(11,12)$. One approach that may elucidate the mechanisms of these functional differences is dissection of the variable (V) region repertoire of Hib PS-specific antibodies. Definition of anti-Hib PS V regions may make it possible to draw $V$ region structure/function correlates. Furthermore, such definition should allow examination of the genetic and environmental factors that influence anti-Hib PS antibody $V$ region repertoire expression.

Serological characterization of anti-Hib PS V regions using polyclonal anti-idiotypic reagents has shown that most individuals express a shared, cross-reactive idiotype (CRI) that comprises a significant portion of the total serum anti-Hib PS antibody $(13,14)$. These findings indicate pauciclonality and lim-

1. Abbreviations used in this paper: CRI, cross-reactive idiotype; DT, diphtheria toxoid; Hib, Haemophilus influenzae type b; Hib PS, capsular polysaccharide of Hib; RABA, radioantigen binding assay. 
ited anti-Hib PS V region heterogeneity. Moreover, the occurrence of a prevalent CRI in unrelated individuals suggests that structurally conserved $\mathrm{V}$ genes may encode a substantial part of the expressed anti-Hib PS repertoire. However, the precision of the idiotypic analyses has been limited by the use of polyclonal anti-CRI reagents, and by the lack of information regarding the structural correlate of the CRI.

A more definitive albeit limited approach to the repertoire has been the molecular characterization of clonally purified anti-Hib PS antibodies from multiple individuals (15-17). These studies have established that anti-Hib PS $V_{H}$ regions are of the $\mathrm{V}_{\mathrm{H}}$ III subgroup, whereas the $\mathrm{V}_{\mathrm{L}}$ regions may be $\mathrm{V} \lambda, \mathrm{V}_{\kappa} \mathrm{I}$, $V_{\kappa}$ III, or most commonly $V_{\kappa}$ II. Remarkably, the $V$ regions of all $\mathrm{V}_{\kappa} \mathrm{II}$ antibodies described to date are derived from the $\mathrm{V}_{\boldsymbol{\kappa}} \mathrm{II}$ gene, A2, and contain little or no somatic mutation in complementarity determining regions (17). However, although sequencing has directly identified particular $\mathrm{V}$ regions, this approach is clearly not amenable for the analysis of serum antibodies in large populations. A combined approach in which anti-idiotypic reagents are used with sequence-defined antiHib PS antibodies, allows for the molecular characterization of CRI determinants, and also provides a convenient and powerful means to examine $V$ region utilization in immune responses to the different Hib PS vaccines in large populations. Here, we report the isolation of a monoclonal antibody (MAb) specific for a CRI expressed by the V $\mathrm{V}_{\kappa}$ IIA2 L chain of anti-Hib PS antibodies. Antibodies bearing this $\mathrm{V}_{\kappa} \mathrm{II}$ CRI are present in most vaccinated infants, children, and adults, and comprise on the average, more than half of the serum antibody to Hib PS.

\section{Methods}

Subjects and vaccinations. Sera from 69 healthy children and 91 healthy adults who were immunized with either one dose of Hib PS vaccine ( 22 children and 66 adults) or with a single dose of a conjugate vaccine consisting of Hib PS coupled to diphtheria toxoid (Hib PS-DT, Connaught Laboratories, Swiftwater, PA) (47 children and 25 adults), were available for study. Serum samples were obtained immediately before immunization and 1-2 mo after immunization. The majority of these subjects have been described in a previous report (14). The principal criteria for inclusion of subjects were the availability of sufficient sera, and the presence of a twofold or greater increase in anti-Hib PS antibody level after vaccination. The only exceptions were four adults, who had $>1 \mu \mathrm{g} / \mathrm{ml}$ of anti-Hib PS antibody in postvaccination sera, but in whom prevaccination sera were not available.

Anti-Hib PS antibody assays. Total antibody to Hib PS was measured using radioantigen binding assays (RABAs) with ${ }^{125}$ I-labeled Hib PS as previously described $(18,19)$, except that precipitates rather than supernatants were counted. Antibody concentrations were determined from a standard curve generated using dilutions of the U. S. Office of Biologics serum reference pool.

Isolation of clonal anti-Hib PS antibodies. Clonal IgG1 and IgG2 anti-Hib PS antibodies were isolated from immune serum of adult $\mathrm{Hib}$ PS vaccine recipients as described in detail (15). Briefly, a series of affinity chromatography steps, first with Hib PS-Sepharose and then with Sepharose-coupled MAbs specific for either IgG1 or IgG2, followed by preparative isoelectric focusing, was used to purify the antibodies to clonality. Clonality of the purified antibodies was confirmed by isoelectric focusing and in most cases by unambiguous amino acid sequence (15-17).

$V$ region amino acid sequencing. Amino-terminal, CDR2, and CDR3 amino acid sequences of $H$ and $L$ chains from clonally purified anti-Hib PS antibodies were determined from isolated $\mathrm{H}$ and $\mathrm{L}$ chains that were either left intact or cleaved at met residues with cyanogen bromide or at trp residues with 2-(2'-nitrophenylsulfenyl)-3-methyl-3'bromoindolenine (17). Amino acid sequences were then compared to known $V_{H}, V_{L}$, and $J_{k}$ protein sequences (20) or to the formal translation products of $\mathrm{V}_{\kappa}$ genes $(17,21)$ for subgroup assignment.

Production of MAb anti-CRI. IgG Anti-Hib PS antibodies were purified from the post-Hib PS vaccination sera of two subjects (20966 and 24604, expressing high levels of CRI as determined with the goat polyclonal anti-CRI reagent) by affinity chromatography (13). Individual BALB/c mice were immunized in several subcutaneous sites with $20 \mu \mathrm{g}$ of subject $20966 \mathrm{IgG}$ anti-Hib PS emulsified in complete Freund's adjuvant. 3 wk later the mice were immunized with the same antigen and dose in incomplete Freund's adjuvant. A third injection of subject 24604 anti-Hib PS in incomplete Freund's adjuvant was given $\sim 6 \mathrm{wk}$ later. Sera were obtained $10-14 \mathrm{~d}$ after the third injection and tested for anti-CRI activity by measuring the ability to inhibit ${ }^{125}$ I-Hib PS binding of subject 24604 anti-Hib PS antibodies (see below). The mouse having the highest serum inhibition titer was immunized intraperitoneally ( $3 \mathrm{wk}$ after the third injection) with a mixture of $10 \mu \mathrm{g}$ of subject 20966 and $10 \mu \mathrm{g}$ of subject 24604 anti-Hib PS in saline. $3 \mathrm{~d}$ later the spleen was taken and used for hybridoma production. Hybridomas were prepared using established methods (22), by fusing spleen cells to the X63-Ag8.653 cell line (23) with 50\% polyethylene glycol. Supernatants were screened for anti-CRI activity by measuring the ability of the supernatant to inhibit ${ }^{125} \mathrm{I}$-Hib PS binding of solid-phase adsorbed anti-Hib PS antibodies (see below). After subcloning by limiting dilution, a hybridoma clone, designated $\mathrm{LuC} 9$ was isolated that secreted MAb capable of inhibiting ${ }^{125}$ I-Hib PS binding by anti-Hib PS antibodies isolated from subjects 20966, 24604, and 21411. MAb was isolated from ascites fluid by ammonium sulfate fractionation and ion exchange chromatography. Alkaline phosphatase (Sigma Chemical Co., St. Louis, MO) was coupled to MAb LuC9 using glutaraldehyde.

Assays for MAb anti-CRI. Hybridoma culture supernatants were screened for anti-CRI activity by measuring the ability of supernatants to inhibit the capture of ${ }^{125}$ I-Hib PS by solid-phase adsorbed anti-Hib PS antibodies. Wells of polyvinyl chloride microtiter plates were coated with subject $21411 \mathrm{IgG}$ anti-Hib PS, $2 \mu \mathrm{g} / \mathrm{ml}$ in PBS, overnight at room temperature. Wells were blocked with BSA, washed with PBSTween, and then incubated with culture supernatant. After $3 \mathrm{~h}$ at $37^{\circ} \mathrm{C}$, half of the supernatant was withdrawn, and ${ }^{125} \mathrm{I}-\mathrm{Hib}$ PS $\left(1 \times 10^{5} \mathrm{cpm} /\right.$ well) was added. Plates were incubated for $3 \mathrm{~h}$ at $37^{\circ} \mathrm{C}$, then washed with PBS-Tween. Wells were excised and bound cpm were determined. Approximately $3 \times 10^{4} \mathrm{cpm}$ of ${ }^{125} \mathrm{I}$-Hib PS was bound in the presence of culture medium alone whereas wells incubated with supernatants having high inhibitory activity had $1-2 \times 10^{3} \mathrm{cpm}$ bound.

An ELISA was used to measure the binding of MAb anti-CRI (LuC9) to human Ig. Microtiter wells were coated with $2 \mu \mathrm{g} / \mathrm{ml}$ of either affinity-purified anti-Hib PS, myeloma proteins (provided by Dr. Hans Spiegelberg, University of California, San Diego), serum $\gamma$-globulin fractions absorbed with Hib PS-Sepharose, human antiHib PS MAbs ED.8 (IgM, $\kappa$ ) or LSF-2 (IgA, $\lambda$ ) (provided by Dr. Penelope Shackelford, Washington University School of Medicine, St. Louis, MO), or human intravenous $\gamma$-globulin (Sandoglobulin, Sandoz Pharmaceuticals Corp., East Hanover, NJ) absorbed with Hib PSSepharose. After incubation at room temperature overnight, the wells were blocked with BSA and washed with PBS-Tween. Dilutions of alkaline phosphatase-conjugated $\mathrm{LuC} 9$ were added to wells and incubated at $37^{\circ} \mathrm{C}$ for $3 \mathrm{~h}$. Wells were then washed, and p-nitrophenyl phosphate $(1 \mathrm{mg} / \mathrm{ml}$ diethanolamine buffer, $\mathrm{pH} 9.5)$ solution was added. Optical density at $405 \mathrm{~nm}$ was determined after $\sim 30 \mathrm{~min}$.

Assay of serum anti-Hib PSCRI expression by anti-CRI inhibition of Hib PS binding. CRI expression by anti-Hib PS antibodies was quantified by measuring the ability of either MAb LuC9 or goat polyclonal anti-CRI, to inhibit Hib PS binding activity in the RABA. This method has been previously described in detail using the polyclonal anti-CRI antibody (14). Briefly, a dilution of human serum or a dilution of human anti-Hib PS MAb, equivalent to an anti-Hib PS antibody concentration of $\sim 200 \mathrm{ng} / \mathrm{ml}$ was mixed with either MAb LuC9 $(60 \mu \mathrm{g} / \mathrm{ml})$ or diluent. The mixtures were incubated for $2 \mathrm{~h}$ at $37^{\circ} \mathrm{C}$ and 
overnight at $4^{\circ} \mathrm{C}$. Each sample was then divided into two aliquots to which ${ }^{125} \mathrm{I}$-Hib PS was added. The samples were incubated for $4 \mathrm{~h}$ at $37^{\circ} \mathrm{C}$ and $1 \mathrm{~h}$ at $4^{\circ} \mathrm{C}$. Saturated ammonium sulfate was added with mixing to a final concentration of $50 \%$ saturation, and after $2 \mathrm{~h}$ at $4^{\circ} \mathrm{C}$, the precipitates were harvested by centrifugation $(16,000 \mathrm{~g})$, washed one time with $50 \%$ saturated ammonium sulfate, and then counted in a dry well $\gamma$ counter. Background counts per minute (i.e., ${ }^{125}$ I-Hib PS precipitated in the presence of MAb LuC9 or diluent alone, which was $<5 \%$ of the total added $\mathrm{cpm}$ ), were subtracted from the respective means of duplicate determinations. Inhibition of Hib PS binding was calculated as previously described (14).

Hib PS inhibition of MAb LuC9 binding to anti-Hib PS antibodies. Wells of microtiter plates were coated with either affinity-purified antiHib PS or human MAb ED.8, then blocked with BSA. Purified Hib PS (provided by Dr. Porter Anderson, University of Rochester School of Medicine, Rochester, NY), diluted in BSA-PBS, or BSA-PBS alone, was added to wells for $3 \mathrm{~h}$ at $37^{\circ} \mathrm{C}$. Half of the solution was withdrawn and alkaline phosphatase-conjugated LuC9 (1:20,000 dilution) was added to the wells. After $3 \mathrm{~h}$ at $37^{\circ} \mathrm{C}$, the wells were washed and $p$-nitrophenyl phosphate in diethanolamine buffer was added. Optical density at $405 \mathrm{~nm}$ was determined at $\sim 30 \mathrm{~min}$. The percent inhibition of MAb LuC9 binding was calculated as previously described (14).

Western blot analysis of MAb Luc 9 binding to anti-Hib PS H and L chains. 3-5 $\mu \mathrm{g}$ of clonally purified anti-Hib PS antibodies were subjected to SDS-PAGE ( $10 \%$ acrylamide) under reducing conditions and electrically blotted onto polyvinylidene difluoride membrane as previously described $(16,17)$. Blots were blocked with $2 \%$ BSA-1\% nonfat dry milk-PBS and subsequently overlayed for $1 \mathrm{~h}$ with a $2 \mu \mathrm{g} / \mathrm{ml} \mathrm{solu-}$ tion of either MAb LuC9 or murine MAb HK-2 which is specific for human $\kappa$ chains. After washing with BSA-PBS, blots were reacted for 1 $h$ with ${ }^{125}$ I-labeled anti-murine $x$ chain antibody (MAb 187), then washed, dried, and exposed to X-Omat film (Eastman Kodak Co., Rochester, NY).

\section{Results}

Isolation of MAb anti-CRI that inhibits Hib PS binding. As described in Methods, a MAb, designated LuC9 (IgG1, $\kappa$ ), was isolated that inhibited the binding of ${ }^{125} \mathrm{I}-\mathrm{Hib}$ PS binding by solid-phase absorbed purified antibodies isolated from three unrelated subjects. The ability of MAb LuC9 to inhibit ${ }^{125} \mathrm{I}-\mathrm{Hib}$ PS binding was examined in the RABA, where a fixed concentration of anti-Hib PS was reacted in solution with varying amounts of LuC9, followed by addition of labeled Hib PS and subsequent determination of binding activity. Fig. 1 shows the results obtained with four different anti-Hib PS samples. LuC9 maximally inhibited by $90 \%$ or greater, the Hib PS binding of subject 2577 serum antibodies and the Hib PS binding of human MAb Ed.8 (IgM, $\kappa)$. Serum anti-Hib PS from subject 24602 was inhibited $20 \%$ by LuC9, and the Hib PS binding of human MAb LSF-2 (IgA, $\lambda$ ) was unaffected by LuC9. These results indicate that the CRI defined by LuC9, hereafter referred to as HibId-1, was expressed by MAb ED.8 and to a variable extent by serum anti-Hib PS antibodies. To quantitate HibId-1 expression with this assay, LuC9 was used at a final concentration of $30 \mu \mathrm{g} / \mathrm{ml}$ in subsequent experiments. In multiple tests, the LuC9 inhibition of Hib PS binding by MAb LSF-2 ranged from $1.1 \%$ to $5.5 \%$ (mean percent inhibition \pm SD $=3.6 \pm 1.4$ ). We chose a value of $\geq 20 \%$ inhibition by $\mathrm{LuC} 9$ as a stringent criterion of HibId-1 positivity. The inhibition of antigen binding by $\mathrm{LuC} 9$ was specific for Hib PS, as LuC9 did not inhibit the binding of anti-DT antibodies in the sera of eight children and eight adults: mean percent inhibition \pm SD of DT binding by LuC9 in ELISA $=-2.1 \pm 5.6 \%$.

To establish that this assay was providing reliable quantita-

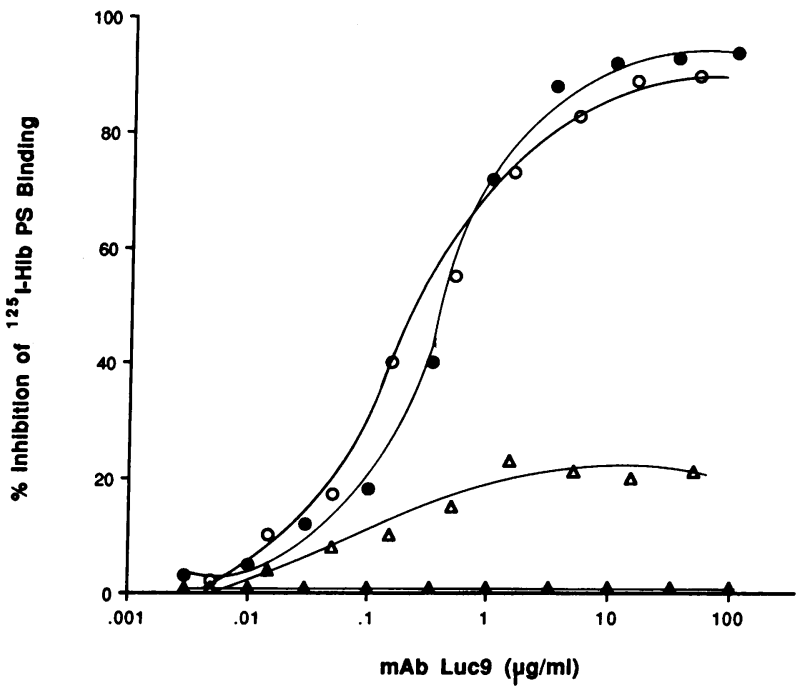

Figure 1. MAb LuC9 inhibition of ${ }^{125} \mathrm{I}-\mathrm{Hib}$ PS binding by MAbs ED.8 $(\bullet)$ and LSF-2 (A) and serum antibodies of subjects $2577(0)$ and $24602(\Delta)$

tive data, we compared the results obtained in this assay with a second method of measuring anti-Hib PS HibId-1 expression. Sera from 11 adults, who had been immunized with Hib PS, were absorbed with either LuC9-agarose or with agarose coupled with an irrelevant murine IgG1, $\kappa$ MAb. The percent specific decrease in the anti-Hib PS antibody level by LuC9-agarose was then determined using the RABA. The HibId-1 antiHib PS values (which ranged from $2 \%$ to $100 \%$ for individual sera) obtained in the inhibition of Hib PS binding assay were in excellent agreement with those obtained by specific absorption $(r=0.99, m=1.02)$. Thus the RABA inhibition assay was suitable for quantifying the fraction of the total serum anti-Hib PS that expressed HibId-1.

$M A b$ LuC 9 binds to some anti-Hib PS antibodies but not to Ig isotypic determinants. An ELISA was used to examine further the specificity of MAb LuC9. Wells of microtiter plates were coated with various Ig preparations then reacted with alkaline phosphatase-coupled LuC9. Fig. 2 shows that LuC9 bound to affinity-purified anti-Hib PS antibodies from three subjects. Consistent with the RABA inhibition results, LuC9 reacted with solid-phase MAb ED.8 (IgM, $\kappa$ ), but not with solid-phase MAb LSF-2 (IgA, $\lambda$ ). LuC9 did not bind to human myeloma proteins representative of all the $\mathrm{H}$ and $\mathrm{L}$ chain isotypes, Ig from three subjects that had been absorbed with Hib PS-agarose, or pooled human IgG (commercial intravenous $\gamma$-globulin) absorbed with Hib PS-agarose.

HibId-1 is related to the Hib PS combining site. The interference of ${ }^{125}$ I-Hib PS binding by LuC9 in the RABA suggested that HibId-1 may be near the combining site. We next determined whether occupancy of the combining site by Hib PS occluded expression of HibId-1. Purified anti-Hib PS antibodies and MAb ED. 8 were absorbed to microtiter wells, and reacted with varying concentrations of Hib PS. The ability of LuC9-alkaline phosphatase to bind was then determined. The results shown in Fig. 3 demonstrate that Hib PS inhibited in a dose-dependent fashion the binding of LuC9 to ED.8 and to purified serum antibodies. HibId-1 expression by MAb ED. 8 was more sensitive to Hib PS inhibition than was HibId-1 expression by purified serum anti-Hib PS antibodies. This find- 


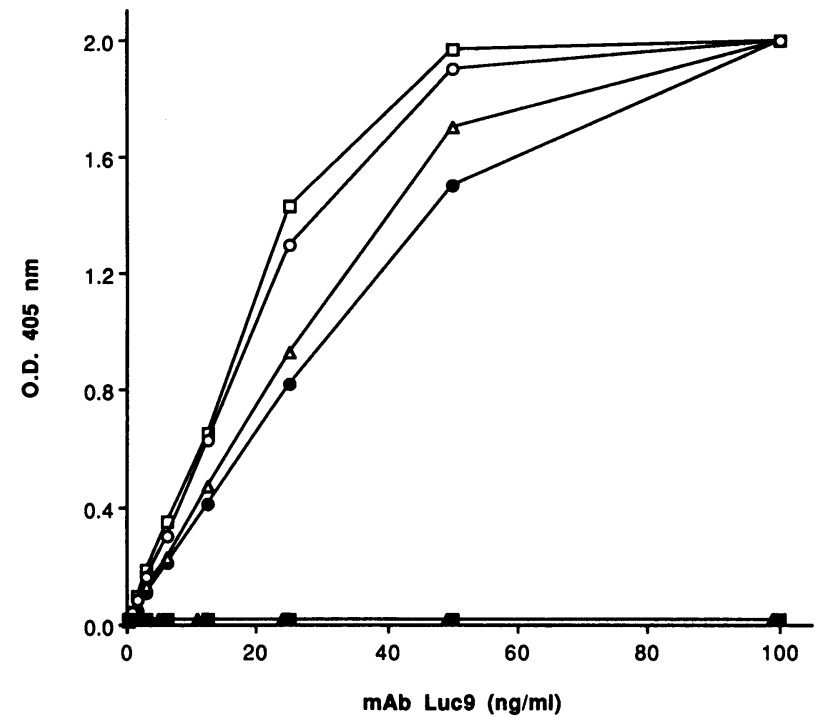

Figure 2. Binding of alkaline phosphatase-conjugated MAb LuC9 to microtiter wells coated with purified anti-Hib PS from subjects 20966

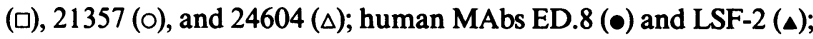
human Ig isotypes-myeloma proteins of the IgG1, 2, 3, 4, IgA1, IgM, and IgD $H$ chain types, and $\kappa$ and $\lambda$ L chains, Hib PS-absorbed Ig from subjects 20966, 21357, and 24604, and Hib PS absorbed human intravenous $\gamma$-globulin ( $(\mathbf{m})$.

ing may be explained by the higher avidity for Hib PS that would be expected for ED.8, an IgM antibody, compared with the serum antibodies which were predominantly IgG.

HibId-1 is a marker of anti-Hib PS antibodies having $V_{\kappa} I I A 2 L$ chains. The expression of HibId-1 by clonally purified anti-Hib PS antibodies, containing $V_{H}$ III chains and various $V_{L}$ chains, was examined using MAb LuC9 inhibition

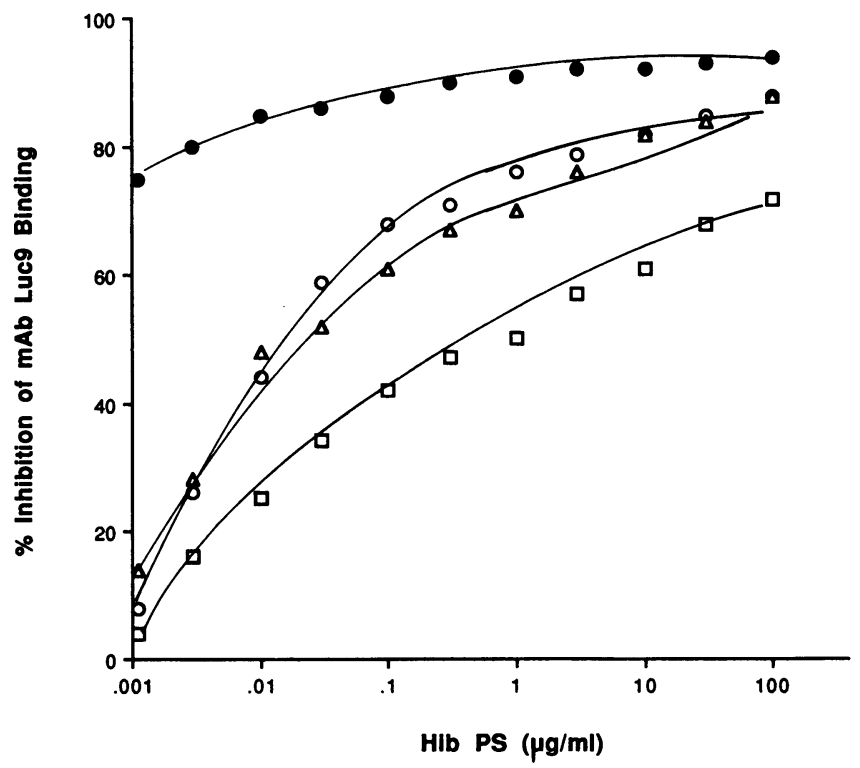

Figure 3. Hib PS inhibition of MAb LuC9-alkaline phosphatase binding to microtiter wells coated with purified anti-Hib PS from subjects $21357(0), 24604(\Delta)$, and $20966(\square)$ and human MAb ED.8 (•). of Hib PS binding in the RABA (Fig. 4). LuC9 did not inhibit Hib PS binding of 10 anti-Hib PS antibodies having either $V \lambda$, $\mathrm{V}_{\kappa} \mathrm{I}$, or $\mathrm{V}_{\boldsymbol{K}} \mathrm{III}$. In contrast, LuC9 inhibited the Hib PS binding of 17 of $17 \mathrm{~V}_{\kappa} \mathrm{II}$ antibodies having either $\mathrm{J}_{\kappa} 1, \mathrm{~J}_{\kappa} 2$, or $\mathrm{J}_{\kappa} 3$ regions. The amino acid sequence analyses of 14 of the 17 antiHib PS antibodies are reported elsewhere $(16,17$, and M. G. Scott, D. L. Crimmins, and M. H. Nahm, manuscript in preparation), and they are all consistent with the $V_{\kappa} I I$ region being derived from the $V_{\kappa} I I A 2$ gene. The sequences in the first and second framework regions and in the CDR 2 and CDR 3 regions were identical to the germ-line $\mathrm{A} 2$ sequence, with the exception of one interchange at residue 94 in one of the L chains (17). Fig. 5 shows the partial amino acid sequences of the remaining three purified anti-Hib PS antibodies, DG1, WG1-II, and DG2b. The sequences are consistent with these antibodies being products of the $\mathrm{A} 2$ gene.

In a previous report we described a goat polyclonal anti-Id reagent, that specifically inhibited Hib PS binding and reacted with a CRI that predominated the serum anti-Hib PS antibodies of most vaccinated subjects (14). In the present study, we found that this polyclonal anti-CRI inhibited the Hib PS binding of only the $\mathrm{V}_{\boldsymbol{K}} \mathrm{IIA} 2$ antibodies (data not shown). This result, as well as others described below, indicate that the polyclonal anti-CRI serum is reacting principally with HibId-1.

HibId-1 is localized to the VKIIA2 L chain. To determine whether HibId-1 was present on isolated $L$ chains, or required pairing of $\mathrm{H}$ and $\mathrm{L}$, we examined the expression of HibId- 1 on free $H$ and $L$ chains from purified clonal anti-Hib PS antibodies after SDS-PAGE under reducing conditions. Fig. 6 shows that LuC9 specifically bound to free $\mathrm{V}_{\kappa}$ IIA $2 \mathrm{~L}$ chains, but did not bind to free $H$ chain or to free $L$ chains of purified $V_{k} I$, $V_{\kappa}$ III, or VkIV subgroup anti-Hib PS antibodies. MAb LuC9 also did not react in Western blot with a $\mathrm{V}_{\boldsymbol{\kappa}} \mathrm{II}$ myeloma protein (data not shown) and an anti-Hib PS antibody expressing a non-A2 $V_{\kappa}$ II $V_{L}$ (M. G. Scott, D. L. Crimmins, D. W. McCourt, K. F. Schäble, R. Thiebe, E.-M. Quenzel, H. G. Zachau, and M. H. Nahm, manuscript in preparation). The identical respective reactivity patterns were observed when blots were tested with the goat polyclonal anti-CRI reagent, again suggesting that both anti-CRI reagents were of similar specificity.

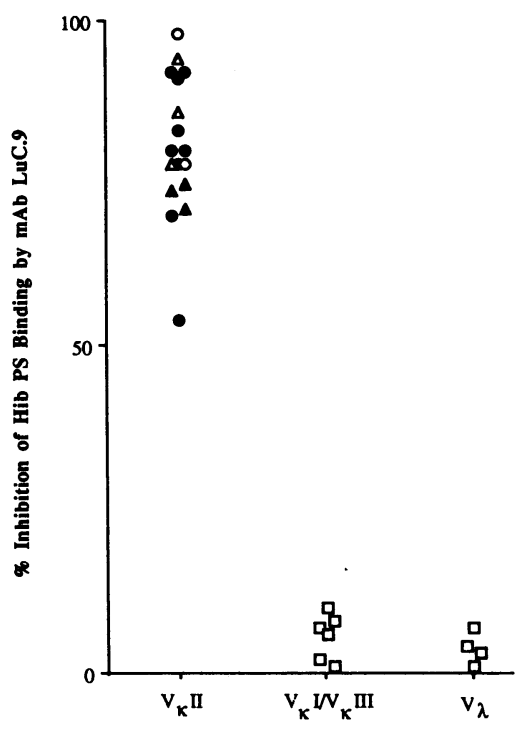

Figure 4. HibId-1 expression by clonal antiHib PS antibodies as determined by MAb LuC9 inhibition of Hib PS binding in RABA. VKII antibodies having $\mathrm{J}_{K} 1$ (๑), $\mathrm{J}_{K} 2(0), \mathrm{J}_{K} 3(\Delta)$, or undetermined $\mathrm{J}_{K}(\Delta)$. 


$\begin{array}{lllllll}1 & 5 & 1 & 1 & 2 & \frac{\mathrm{CDR} 1}{22 \mathrm{abcd}} \\ & & & 0 & 5 & 0 & 57\end{array}$

"A2" DIVMTQTPLSLSVTPGQPASISCKSSQSLLH

DG1

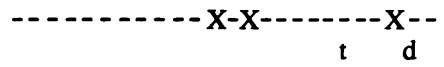

WG1-II

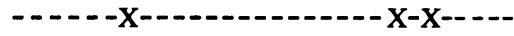

DG2b

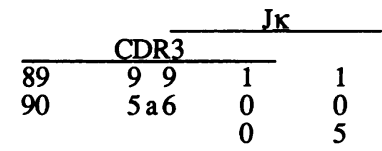

MQSIQLP

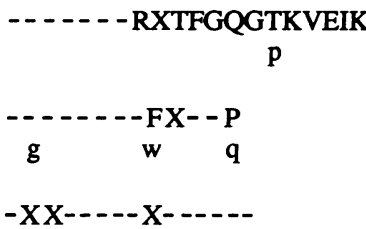

Figure 5. Amino-terminal and CDR3 formal translation product of $V_{\kappa} I I A 2$ gene (17), upper sequence, and amino acid sequences of three purified anti-Hib PS antibodies, DG-1, WG1-II, and DG2b.

JK1 (3) Dayhoff notation and Kabat numbering systems are used (21). Underlined residues indicate that they are either unique to $A 2$ or occur together only in A2 (17).

Dashes indicate identity to A2 sequence. Lower case let-

ters indicate uncertain designation or minor contaminating residues. $\mathrm{X}$ indicates no residue identified. $\mathrm{X}$ at position 23 is likely an invariant cysteine not identifiable by our sequencing method. Parentheses ( ) refer to possible $\mathrm{J}_{\kappa}$ designation based on minor residues identified in $\mathrm{J}_{\kappa}$ region.

HibId-1 is prevalent and predominates the serum anti-Hib $P S$ induced by vaccination. Individual postvaccination sera were analyzed for anti-Hib PS HibId-1 expression using RABA inhibition by LuC9 (Fig. 7). $86 \%$ of adults and $83 \%$ of children, were positive for HibId-1 anti-Hib PS after vaccination with Hib PS, i.e., subjects having $\geq 20 \%$ of their serum anti-Hib PS antibody activity inhibitable by MAb LuC9. Immunization with Hib PS-DT gave similarly high frequencies of HibId-1 positivity in infants $(71 \%)$, older children $(77 \%)$, and adults $(96 \%)$. The fraction of the total serum anti-Hib PS that expressed HibId-1 centered around $60 \%$ for all age groups and for both vaccines. There were no significant differences between the mean percent LuC9 inhibition values for the five groups $(P>0.4$ by $t$ test), nor was there any correlation between the log of the anti-Hib PS antibody concentration and the LuC9 inhibition value in Hib PS postvaccination sera of children $(r=-0.148, P>0.5)$ or adults $(r=0.08, P>0.5)$.

A comparison was made between the HibId-1 anti-Hib PS levels of 88 post-Hib PS vaccination sera from children and adults as determined by LuC9 inhibition and the goat polyclonal anti-CRI inhibition of Hib PS binding in the RABA. The paired inhibition values were highly correlated $(r=0.9, m$ $=1.04$ ). Thus in keeping with the other comparisons, the dominant Id recognized by the polyclonal anti-CRI reagent appears to be the same or similar to HibId-1, defined by LuC9.

Differences in HibId-1 expression between pre-and postvaccination antibodies. The anti-Hib PS HibId-1 levels were compared between sera obtained from adults before and after vaccination with Hib PS (Fig. 8). Of 62 subjects, 32 showed a $>20 \%$ increase in their HibId-1 anti-Hib PS level after vaccination. In contrast, only two subjects showed greater than a $20 \%$ decrease in serum HibId-1 anti-Hib PS after vaccination. This relative increase in HibId-1 after vaccination was also apparent when the data were expressed as percentage of subjects positive for HibId-1 antibodies. Before immunization, $56 \%$ of subjects were HibId-1-positive, i.e., had $\geq 20 \%$ of their serum anti-Hib

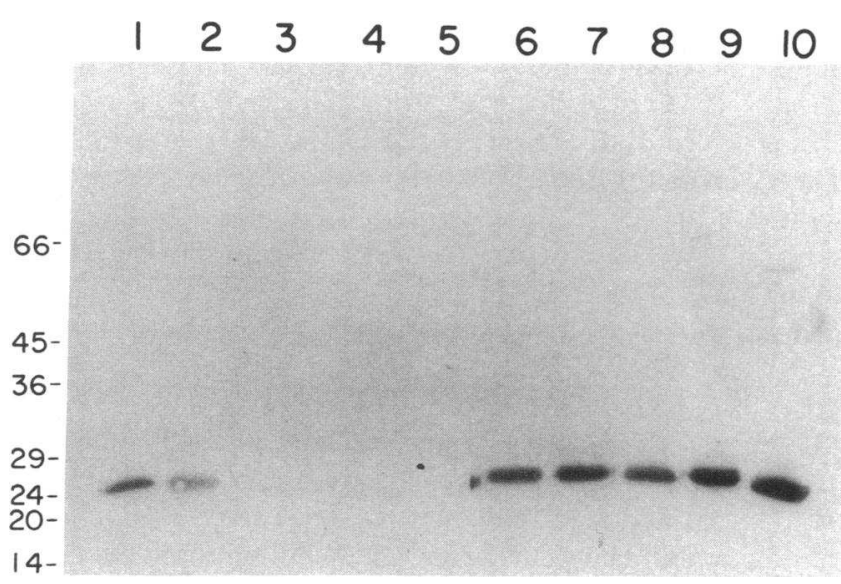

Figure 6. HibId-1 is localized to $\mathrm{V}_{\boldsymbol{K}} \mathrm{II}$ chains. Blots of SDS-PAGE separated $\mathrm{H}$ and $\mathrm{L}$ chains from clonally purified anti-Hib PS antibodies, were reacted with either MAb LuC9 (lanes 1-5), or MAb HK-2 (specific for human $\kappa$ chains) (lanes 6-10). Blots were then reacted with ${ }^{125} \mathrm{I}$-labeled anti-mouse $\kappa$, followed by autoradiography: Lanes 1 and 6, VkIIA2 (B-G2); lanes 2 and 7, VkIIA2 (C-G2); lanes 3 and $8, \mathrm{~V}_{\kappa} \mathrm{I}$ (H-G2); lanes 4 and 9, $\mathrm{V}_{\kappa} \mathrm{IV}$ (DT3-G2); lanes 5 and 10, VkIII (DT12-G1).

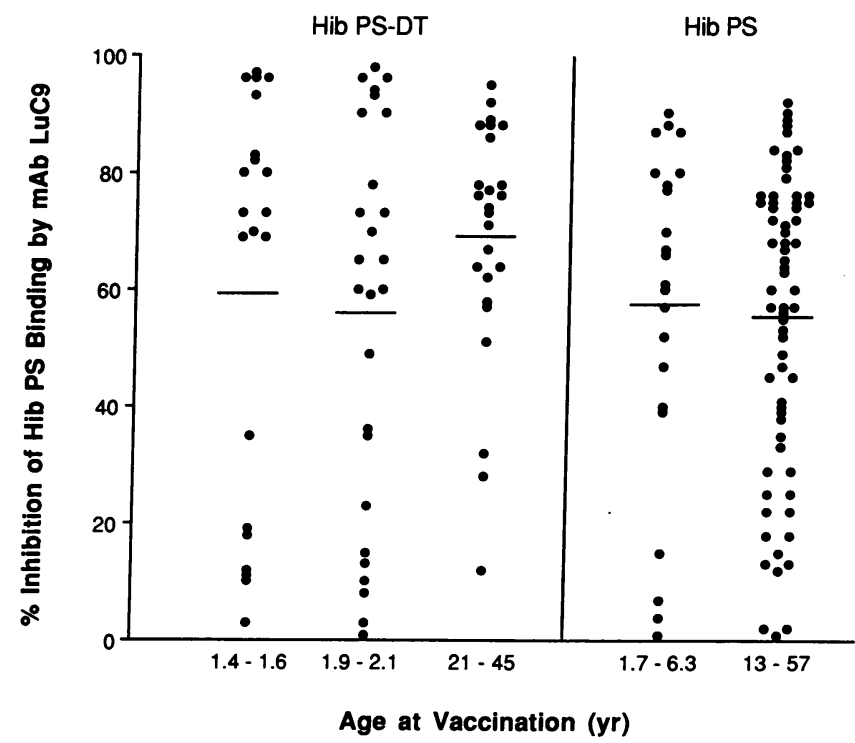

Figure 7. Hibld-1 expression by anti-Hib PS antibodies after vaccination with either Hib PS or Hib PS-DT. Each circle represents the percent MAb LuC9 inhibition of ${ }^{125} \mathrm{I}-\mathrm{Hib}$ PS binding of an individual serum. Mear percent inhibition of each group is indicated by the horizontal bar. 


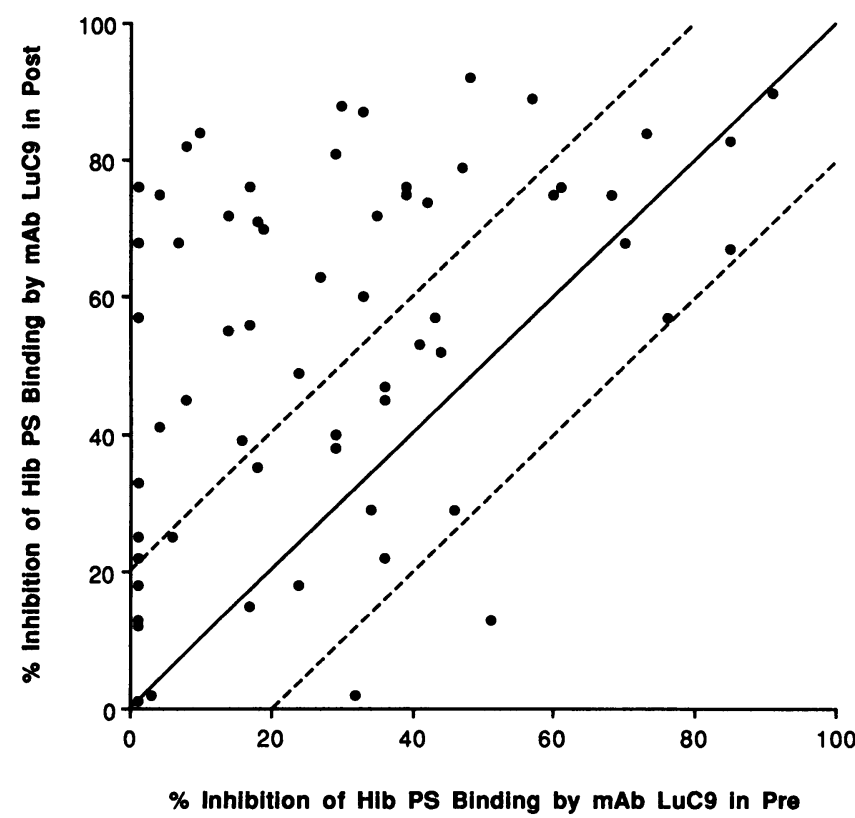

Figure 8. HibId-1 anti-Hib PS expression in pre- and post-Hib PS vaccination sera of 62 adults. Each circle represents the percent MAb LuC9 inhibition of ${ }^{125} \mathrm{I}$-Hib PS binding in individual paired sera. The solid diagonal line represents no change in the relative proportion of serum HibId-1-positive anti-Hib PS antibody after vaccination. The dotted lines represent a $20 \%$ increase or decrease in the serum HibId-1 anti-Hib PS levels after vaccination.

PS activity inhibitable by MAb LuC9, whereas after immunization with Hib PS, $85 \%$ of subjects were positive for HibId-1 anti-Hib PS antibody.

\section{Discussion}

We have shown previously that human antibodies to Hib PS exhibit a high degree of idiotypic cross-reactivity $(13,14)$, and commonly use a conserved $\mathrm{V}_{\kappa} \mathrm{II} \mathrm{V}_{\mathrm{L}}$ region, encoded by the $\mathrm{A} 2$ gene and containing little or no somatic mutation (17). We suspected that these two characteristics may be related because sharing of Id frequently indicates structural homology and similar genetic origin. The data presented here demonstrate that this relationship is indeed the case. A monoclonal antibody, as well as the previously described goat polyclonal antiserum (14), were found to react with a CRI, designated HibId-1, expressed by anti-Hib PS antibodies having $V_{\kappa}$ II $L$ chains derived from the $\mathrm{A} 2$ gene.

Western blot analysis showed that the HibId-1 determinant was present on the isolated $V_{\kappa}$ IIA2 chain of anti-Hib PS antibodies, indicating that quaternary interactions with the $\mathrm{H}$ chain are not required for Id expression. No association was found between HibId-1 expression and the $\mathrm{J}_{K}$ genes expressed by these $\mathrm{A}$ 2-encoded $\mathrm{V}$ regions, suggesting that the $\mathrm{J}$ region is not part of the Id determinant. However, an arginine at position $95 \mathrm{a}$, created by the joining of $\mathrm{V}$ and $\mathrm{J}$, occurs in all of the VkIIA2 regions sequenced thus far (17), and therefore the structural determinants of HibId-1 may include sequences in the A2 $\mathrm{V}$ region in association with this rearrangement residue. Previously, we have postulated that this invariant arginine may be essential for binding the negatively charged Hib PS (17). Inter- estingly, murine antibodies specific for azophenylarsonate also have an invariant arginine at the V-J joint (24), and chain recombination experiments show that this residue is essential for arsonate binding (25). The finding that HibId-1 expression was inhibited by occupancy of the combining site with Hib PS raises the possibility that the HibId-1 determinant may be near putative contact residues such as the invariant arginine.

The availability of anti-idiotypic probes of defined specificity permitted us to examine the expression of the $\mathrm{V}_{\kappa}$ IIA2 region in the vaccine-induced responses of a large number of subjects. The principal finding was that anti-Hib PS antibodies bearing HibId-1/VkIIA2 were prevalent in the responses induced by both free and DT-conjugated Hib PS, and comprised on the average $60 \%$ of the total serum antibody. Neither the frequency of expression nor the predominance of Hibld-1 among Hib PS-DT-induced antibodies was significantly different between infants, older children and adults. Previously, we observed that anti-Hib PS antibodies induced in 2-3 mo old infants by immunization with Hib PS conjugated to the outer membrane protein of Neisseria meningitidis, expressed high levels of HibId-1 as detected using the polyclonal anti-CRI reagent, that predominantly recognizes HibId-1 (14). Taken together, these findings indicate that anti-Hib PS antibodies having $V_{\kappa}$ IIA2 $L$ chains dominate both the infant and adult repertoires induced by vaccination. While the recurrence of a particular germ-line encoded $\mathrm{V}$ region may appear to be surprising in an outbred human population with high potential for generating antibody diversity, it is consistent with the expression of conserved $\mathrm{V}$ regions by human autoantibodies (26), and the dominant utilization of a single $\mathrm{V}_{\mathrm{L}}$ in the murine antibody response to phosphorylcholine (27).

The predominance of HibId-1/VkIIA2 antibodies in individual responses to Hib PS, demonstrates the restricted heterogeneity of anti-Hib PS V regions, and is in agreement with isoelectric focusing studies showing that individual anti-Hib PS clonotypes are frequently oligoclonal and may be shared between subjects $(20,28)$. Nevertheless, it is important to note that considerable diversity is also present in anti-Hib PS antibodies. Not only can the anti-Hib PS V $k$ IIA2 region associate with several different $J$ regions and thereby generate variability in the third CDR of these antibodies, but anti-Hib PS antibodies may also contain $V \lambda, V_{\kappa} I$, non $A 2-V_{\kappa} I I, V_{\kappa} I I I$, and $V_{\kappa} I V$ chains (16, and $M$. S. Scott et al, manuscript in preparation). The $V_{H}$ chains of anti-Hib PS antibodies have just begun to be scrutinized. They are most commonly restricted to the $\mathrm{V}_{\mathrm{H}} \mathrm{III}$ subgroup (16), and by serological analysis they appear related to the $\mathrm{V}_{\mathrm{H}}$ III 30P1 and 20P1 genes (29). Nucleic acid sequence analysis shows that four of five anti-Hib PS hybridomas use a $\mathrm{V}_{\mathrm{H}}$ III region having $83-94 \%$ homology with the 9.1 (20P1) gene (30), in conjunction with a variety of $D$ and $J$ elements (31). Thus, despite the consistent usage and predominance of the $V_{\kappa}$ IIA2 gene product, there is significant $V_{L}$ diversity in the non- $\mathrm{A} 2$ antibodies, as well as $\mathrm{V}_{\mathrm{H}}$ heterogeneity. It will be important to ultimately determine whether this molecular heterogeneity correlates with the observed functional heterogeneity among anti-Hib PS antibodies $(11,12)$.

The representation of HibId-1 in the prevaccination or socalled natural anti-Hib PS antibody populations was less than that seen with the vaccine-induced antibodies. The lower expression in prevaccination sera was apparent in both the frequency of HibId-1-positive subjects as well as in the fraction of 
the total serum antibody that expressed HibId-1. Natural antibody to Hib PS is probably induced by both Hib colonization and by bacteria having Hib PS cross-reactive antigens, such as Escherichia coli $\mathrm{K} 100(32,33)$. K100 cross-reactive anti-Hib PS antibodies probably do not express HibId-1 since a previous study showed that K100-reactive antibodies had either $\mathrm{V} \lambda$, $\mathrm{V}_{\kappa} \mathrm{I}$, or $\mathrm{V}_{\kappa} \mathrm{III}$ chains but not $\mathrm{V}_{\kappa} \mathrm{II}(15)$. Thus, mucosal stimulation by $E$. coli or other Hib PS cross-reactive organisms could skew the natural, prevaccine repertoire toward HibId-1/ $V_{\kappa}$ IIA2 negativity. Upon vaccination, HibId-1 anti-Hib PS antibodies appeared to be preferentially induced in the majority of subjects. One mechanism that could account for this preference is that B cells expressing HibId-1/VKIIA2, have higher affinity for Hib PS than HibId-1-negative B cells and therefore are selectively activated. The modest net increases in avidity that have been observed in adults after immunization with $\mathrm{Hib}$ PS may reflect this process (34).

In addition to somatic selection of the $V_{\kappa}$ IIA2 region, there is the question of evolutionary selection. The VKIIA2 gene is likely to be widely present in the human genome based upon the high frequency of HibId-1 expression in individual antiHib PS responses. Perhaps the V $\kappa$ IIA2 gene has been selected and maintained in the germ-line because it confers optimal protection against Hib. Such a mechanism has been proposed as an explanation for the conservation of murine germ-line $\mathrm{V}$ region genes that encode antibodies reactive with bacterial antigens $(35,36)$. However, HibId-1/V $\kappa$ IIA2-negative antibodies comprise on the average $\sim 40 \%$ of the vaccine-induced serum anti-Hib PS antibody, and some individuals (probably $10 \%$ or less) respond to vaccination with little or no HibId-1 anti-Hib PS antibody. HibId-1-negative antibodies are probably protective since in vitro they activate complement-mediated bactericidal activity (11). Thus, it is questionable whether $V_{\kappa}$ IIA2 antibodies are necessarily more protective than $V_{\kappa}$ IIA2-negative antibodies. An alternative theory for the evolutionary conservation of the $V_{K}$ IIA2 gene is that by encoding HibId-1 antibodies, it becomes part of a functional idiotypic network, and therefore has been selected based upon its idiotopic rather than its paratopic fitness $(37,38)$.

A hallmark of the antibody response to Hib PS is age dependence. The ability to produce antibodies in response to immunization with the free polysaccharide does not fully mature until $\sim 6$ y of age $(39,40)$. The mechanism(s) of age-related unresponsiveness to free Hib PS is poorly understood. Absence of specific $B$ cells cannot be the explanation as shown by the immunogenicity of the conjugate vaccines in infants. Although age-related rearrangement and expression of Ig genes is known to occur (41-44) and has been suggested as a possible mechanism controlling repertoire maturation, the data presented here demonstrate that the $V_{\kappa}$ IIA2 gene product is functionally expressed in infants immunized with conjugate vaccine. In addition, as noted above, anti-Hib PS $\mathrm{V}_{\mathbf{H}}$ regions may derive from the I.9/20P1 and 30P1 related genes, and these genes are known to be expressed in the fetal repertoire (45). Therefore, age-related unresponsiveness to free Hib PS does not appear to be related to the inability to generate the appropriate $\mathrm{V}$ regions.

\section{Acknowledgments}

The authors are grateful to Penelope Shackelford for sharing the human MAbs, and to Gregg Silverman for critical comments.
This work was supported by grants AI-25008 (Dr. Lucas) and AI17962 (Dr. Granoff) from the National Institutes of Allergy and Infectious Diseases, and grant 1902 from the Council for Tobacco Research (Dr. Nahm).

\section{References}

1. Robbins, J., R. Schneerson, and M. Pittman. 1984. Haemophilus influenzae b infections. In Bacterial Vaccines. R. Germanier, editor. Academic Press, Inc., Orlando, FL. 289-316.

2. Moxon, E., R, and R. Rappuoli. 1990. Haemophilus influenzae infections and whooping cough. Lancet. 335:1324-1329.

3. Granoff, D. M., and K. L. Cates. 1985. Haemophilus influenzae type b polysaccharide vaccines (medical progress). J. Pediatr. 107:330-336.

4. Schneerson, R., O. Barrera, A. Sutton, and J. B. Robbins. 1980. Preparation, characterization, and immunogenicity of Haemophilus influenzae type b polysaccharide-protein conjugates. J. Exp. Med. 152:361-376.

5. Weinberg, G. A., and D. M. Granoff. 1988. Polysaccharide-protein conjugate vaccines for the prevention of Haemophilus influenzae type b disease. $J$. Pediatr. 113:621-631.

6. Anderson, P., M. Pichichero, K. Edwards, C. R. Porch, and R. Insel. 1987. Priming and induction of Haemophilus influenzae type b antibodies in early infancy by Dpo20, an oligosaccharide-protein conjugate vaccine. J. Pediatr. 111:644-650.

7. Einhorn, M. S., G. A. Weinberg, E. L. Anderson, P. D. Granoff, and D. M. Granoff. 1986. Immunogenicity in infants of Haemophilus influenzae type b polysaccharide in a conjugate vaccine with Neisseria meningitidis outer-membrane protein. Lancet. ii:299-302.

8. Granoff, D. M., A. Chacko, K. R. Lottenbach, and K. E. Sheetz. 1989. Immunogenicity of Haemophilus influenzae type b polysaccharide-outer membrane protein conjugate vaccine in patients who acquired Haemophilus disease despite previous vaccination with type $\mathrm{b}$ polysaccharide vaccine. J. Pediatr. 114:925-933.

9. Ward, J., G. Brenneman, G. W. Letson, W. L. Heyward, and the Alaska $H$. influenzae Vaccine Study Group. 1990. Limited efficacy of a Haemophilus influenzae type b conjugate vaccine in Alaska native infants. $N$. Engl. J. Med. 323:1393-1401.

10. Siber, G. R., M. Santosham, G. R. Reid, C. Thompson, J. Almeido-Hill, A. Morell, G. deLange, J. K. Ketcham, and E. H. Callahan. 1990. Impaired antibody response to Haemophilus influenzae type b polysaccharide and low IgG2 and IgG4 concentrations in Apache children. N. Engl. J. Med. 323:13871392.

11. Amir, J., M. G. Scott, M. H. Nahm, and D. M. Granoff. 1990. Bactericidal and opsonic activity of IgG1 and IgG2 anticapsular antibodies to Haemophilus influenzae type b. J. Infect. Dis. 162:163-171.

12. Amir, J., X. Liang, and D. M. Granoff. 1990. Variability in the functional activity of vaccine-induced antibody to Haemophilus influenzae type b. Pediatr. Res. 27:358-364.

13. Lucas, A. H. 1988. Expression of crossreactive idiotypes by human antibodies specific for the capsular polysaccharide of Haemophilus influenzae type b. J. Clin. Invest. 81:480-486.

14. Lucas, A. H., and D. M. Granoff. 1990. A major crossreactive idiotype associated with human antibodies to the Haemophilus influenzae b polysaccharide: expression in relation to age and IgG subclass. J. Clin. Invest. 85:1 158-1166.

15. Tarrand, J., M. S. Scott, P. E. Takes, and M. H. Nahm. 1989. Clonal characterization of the human IgG antibody repertoire to Haemophilus influenzae type $\mathrm{b}$ polysaccharide $\mathrm{I}$ : demonstration of three types of $\mathrm{V}$ regions and their association with $\mathrm{H}$ and $\mathrm{L}$ chain isotypes. J. Immunol. 142:2519-2526.

16. Scott, M. G., J. J. Tarrand, D. L. Crimmins, D. W. McCourt, N. R. Siegel, C. E. Smith, and M. H. Nahm. 1989. Clonal characterization of the human IgG antibody repertoire to Haemophilus influenzae type b polysaccharide II. IgG antibodies contain VH genes from a single family and VL genes from at least four VL families. J. Immunol. 143:293-298.

17. Scott, M. G., D. L. Crimmins, D. W. McCourt, I. Zocher, R. Thiebe, H. G. Zachau, and M. H. Nahm. 1989. Clonal characterization of the human antibody repertoire to Haemophilus influenzae type b polysaccharide III: a single $V_{\kappa}$ II gene and one of several $\mathrm{J}_{K}$ genes are joined by an invariant arginine to form the most common L chain V region. J. Immunol. 143:4110-4116.

18. Robbins, J. B., J. C. Parke, R. Schneerson, and J. K. Whisnant. 1973 Quantitative measurement of "natural" and immunization-induced Haemophilus influenzae type b capsular polysaccharide antibodies. Pediatr. Res. 7:103110.

19. Granoff, D. M., P. G. Shackelford, J. P. Pandey, and E. G. Boies. 1986. Antibody responses to Haemophilus influenzae type b polysaccharide vaccine in relation to $\mathrm{Km}(1)$ and $\mathrm{G} 2 \mathrm{~m}(23)$ immunoglobulin allotypes. J. Infect. Dis. 154:257-264.

20. Kabat, E. A., T. T. Wu, M. Reid-Miller, H. M. Perry, and K. S. Gottes- 
man. 1987. Sequences of Proteins of Immunological Interest, 4th edition. U. S. Department of Health and Human Services, National Institutes of Health, Bethesda, MD.

21. Meindl, A., H. G. Klobeck, R. Ohnheiser, and H. G. Zachau. 1990. The $\mathrm{V}_{\kappa}$ repertoire in the human germline. Eur. J. Immunol. 20:1855-1863.

22. Fazekas de St. Groth, S., and D. Scheidegger. 1980. Production of monoclonal antibodies: strategy and tactics. J. Immunol. Methods. 47:129-144.

23. Kearney, J. F., A. Radbruch, B. Liesengang, and K. Rajewsky. 1979. A new mouse myeloma cell line that has lost Ig expression but permits construction of antibody-secreting hybrid cell lines. J. Immunol. 123:1548-1550.

24. Siegelman, M., and J. D. Capra. 1981. Complete amino acid sequence of light chain variable regions derived from five monoclonal anti-p-azophenylarsonate antibodies differing with respect to cross-reactive idiotype. Proc. Natl. Acad. Sci. USA. 78:7679-7683.

25. Jeske, D. J., J. Jarvis, C. Milstein, and J. D. Capra. 1984. Junctional diversity is essential to antibody activity. J. Immunol. 133:1090-1092.

26. Carson, D. A., P. P. Chen, and T. J. Kipps, Editors. 1990. Idiotypes in Biology and Medicine. S. Karger, Basel. 212 pp.

27. Perlmutter, R. M., S. T. Crews, R. Douglas, G. Sorenson, N. Johnson, N Nivera, P. J. Gearhart, and L. Hood. 1984. The generation of diversity of phosphorylcholine-binding antibodies. Adv. Immunol. 35:1-37.

28. Insel, R. A., A. Kittelberger, and P. W. Anderson. 1985. Isoelectric focusing of human antibodies to the Haemophilus influenzae b capsular polysaccharide: restricted and identical spectrotypes in adults. J. Immunol. 135:2810-2816.

29. Silverman, G. J., and A. H. Lucas. 1991. Variable region diversity in human circulating antibodies specific for the capsular polysaccharide of Haemophilus influenzae type b: preferential usage of two types of $\mathrm{V}_{\mathrm{H}} 3$ heavy chains. $J$ Clin. Invest. 88:911-920.

30. Berman, J. E., S. J. Mellis, R. Pollock, C. L. Smith, H. Suh, B. Heinke, C Kowal, U. Surti, L. Chess, C. R. Cantor, et al. 1988. Content and organization of the human Ig VH locus: definition of three new VH families and linkage to the CH locus. EMBO (Eur. Mol. Biol. Organ.) J 7:727-738.

31. Adderson, E. E., P. G. Shackelford, A. Quinn, and W. L. Carroll. 1991. Restricted IgH chain V gene usage in the human antibody response to Haemophilus influenzae type b capsular polysaccharide. J. Immunol. 147:1667-1674.

32. Schneerson, R., M. Bradshaw, J. K. Whisnant, R. L. Myerowitz, J. C. Parke, Jr., and J. B. Robbins. 1972. An Escherichia coli antigen cross-reactive with the capsular polysaccharide of Haemophilus influenzae type b: occurrence among known serotypes, and immunochemical and biological properties of $E$. coli antisera towards $H$. influenzae type b. J. Immunol. 108:1551-1562.

33. Insel, R. A., and P. W. Anderson. 1982. Cross-reactivity with Escherichia coli $\mathrm{K} 100$ in the human serum anticapsular antibody response to Haemophilus influenzae type b. J. Immunol. 128:1267-1270.

34. Griswold, W. R., A. H. Lucas, Bastian, J. F., and G. Garcia. 1989. Functional affinity of antibody to Haemophilus influenzae b polysaccharide. J. Infect. Dis. 159:1083-1087.

35. Cohn, M. 1986. Idiotype network views of immune regulation: for whom the bell tolls. In Idiotypes. M. Reichlin and J. D. Capra, editors. Academic Press Inc., Orlando, FL. 321-399.

36. Briles, D. E., C. Forman, S. Hudak, and J. L. Claflin. 1982. Anti-phosphorylcholine antibodies of the T15 idiotype are optimally protective against Streptococcus pneumoniae. J. Exp. Med. 156:1177-1185.

37. Holmberg, D., A. Andersson, L. Carlsson, and S. Forsgren. 1989. Establishment and functional implications of B-cell connectivity. Immunol. Rev. 110:89-103.

38. Kearney, J. F., and M. Vakil. 1986. Idiotype-directed interactions during ontogeny play a major role in the establishment of the adult $B$ cell repertoire. Immunol. Rev. 94:39-49.

39. Smith, D. H., G. Peter, D. L. Ingram, A. L. Harding, and P. Anderson. 1973. Responses of children immunized with the capsular polysaccharide of Haemophilus influenzae type b. Pediatrics. 52:637-644.

40. Granoff, D. M., and R. S. Munson, Jr. 1986. Prospects for the prevention of $H$. influenzae type b disease by immunization. J. Infect. Dis. 153:448-461.

41. Alt, F. W., T. K. Blackwell, and G. D. Yancopoulos. 1987. Development of the primary antibody repertoire. Science (Wash. DC). 238:1079-1087.

42. Schroeder, H. W., J. L. Hillson, and R. M. Perlmutter. 1987. Early restriction of the human antibody repertoire. Science (Wash. DC). 238:791-793.

43. Korsmeyer, S. J., P. A. Heiter, S. O. Sharrow, C. K. Goldman, P. Leder, and T. A. Waldman. 1982. Normal human B cells display ordered light chain rearrangements and deletions. J. Exp. Med. 156:975-985.

44. Kaushik, A., D. H. Schultze, C. Bona, and G. Kelsoe. 1989. Murine $V_{\kappa}$ gene expression does not follow the VH paradigm. J. Exp. Med. 169:1859-1864.

45. Schroeder, H. W., Jr., and J. Y. Wang. 1990. Preferential utilization of conserved immunoglobulin heavy chain variable gene segments during fetal life. Proc. Natl. Acad. Sci. USA. 87:6146-6150. 